\title{
SONIFYING STOCHASTIC WALKS ON BIOMOLECULAR ENERGY LANDSCAPES
}

\author{
Robert E. Arbon ${ }^{1 *}$, Alex J. Jones ${ }^{1,2,3, *}$, Lars A. Bratholm ${ }^{1,4}$, Tom Mitchell ${ }^{3}$, and David R. Glowacki ${ }^{1,2}$ \\ * These authors contributed equally to this manuscript \\ ${ }^{1}$ School of Chemistry, University of Bristol, Bristol, BS8 1TS, UK \\ ${ }^{2}$ Dept. of Computer Science, University of Bristol, Bristol, BS8 1UB, UK \\ ${ }^{3}$ Dept. of Computer Science and Creative Technologies, University of the West of England, Bristol, BS16 1QY, UK \\ ${ }^{4}$ School of Mathematics, University of Bristol, University Walk, Bristol, BS8 1TW, UK \\ \{robert.arbon, alex.j.jones, lars.bratholm, glowacki\}@bristol.ac.uk \\ tom.mitchell@uwe.ac.uk
}

\begin{abstract}
Translating the complex, multi-dimensional data produced by simulations of biomolecules into an intelligible form is a major challenge in computational chemistry and biology. The so-called "free energy landscape" is amongst the most fundamental concepts used by scientists to understand both static and dynamic properties of biomolecular systems. In this paper we use Markov models to design a strategy for mapping features of this landscape to sonic parameters, for use in conjunction with visual display techniques such as structural animations and free energy diagrams. This allows for concurrent visual display of the physical configuration of a biomolecule and auditory display of characteristics of the corresponding free energy landscape. The resulting sonification provides information about the relative free energy features of a given configuration including its stability.
\end{abstract}

\section{INTRODUCTION}

\subsection{Project context}

Richard Feynman famously stated [1] that "everything that living things do can be understood in terms of the jigglings and wigglings of atoms". A complete understanding of how these atomic jigglings and wigglings give rise to the structure, dynamics and function of biomolecules remains an outstanding scientific challenge with implications across a wide range of disciplines. For example, dynamical processes like protein folding are implicated in neurological diseases (e.g. Alzheimer's) [2] and conformational changes in enzymes are linked to their biological function [3].

Computer simulation is an important tool to understand biomolecular dynamics because of its ability to reveal chemical information at the atomic level with a high degree of temporal and spatial resolution [4] 5]. Its popularity is associated with three developments: accurate and computationally efficient ways of modeling the interactions between atoms (the atomic 'force-field')[6], the increasing availability of highly parallel computer architectures such as general purpose graphical processing units (GP-GPUs) [7], and a variety of user friendly software packages which exploit both these developments [8] 9]. This has enabled the study of bigger systems at longer timescales, moving the dynamics of biomolecular systems into the 'big-data' era [10].

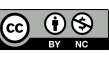

This work is licensed under Creative Commons Attribution Non Commercial 4.0 International License. The full terms of the License are available at http://creativecommons.org/licenses/by-nc/4.0
Extracting scientific information from the output of computer simulations is difficult owing to the quantity of data available. Output from molecular dynamics (MD) simulations [11] include time series of atomic positions (known as trajectories) and associated data (e.g., system energy, volume, pressure, etc.). Making sense of this data requires reducing the dimensionality by removing irrelevant features and producing an accurate but understandable model of the process being investigated. Analysing trajectories is commonly performed through visual display using animations of the molecules (often with atoms rendered as balls and chemical bonds as sticks). However, there is typically far too much data for a researcher to understand. Dimensionality reduction is achieved by only displaying certain atoms while features of the data can be calculated and mapped to visual aesthetics. For example, common structural motifs in proteins, such as alpha-helices, can be drawn as a cartoon helix on top of the molecular structure to highlight their presence. A particularly important feature of the system is its free energy. Any given molecular configuration has an associated free energy, from which several important properties can be calculated [12]. Often described as a "landscape", free energy is commonly represented as a topographical contour map. "Mapping" the free energy landscape of biomolecular systems remains a significant challenge. Nevertheless, understanding how a molecule's 3D structure relates to its free energy landscape is crucial for scientists to gain an understanding of biomolecular dynamics. The software package MolPX [13] has attempted this by linking two separate visual objects - molecular animations and free energy diagrams. However this strategy has two drawbacks: (1) the free energy landscape is limited to two dimensions and (2) the researcher's focus is split across the two visual objects. Display of higher dimensional landscapes is possible by combinations of two dimensional projections but this only exacerbates problem (2). Sonification has the ability to overcome problems with displaying high dimensional free energy landscapes: the topography and important features of the landscape can be heard concurrently with visual structural information. However, creating a sonic representation of features of the free energy landscape presents a number of technical and design related challenges which are explored in this paper.

\subsection{Sonification techniques for molecular data}

Two major techniques for approaching an auditory display challenge are model-based and parameter mapping sonification (PMSon). Model-based techniques aim to transform a dataset into a dynamic model, which one can interact with and aurally examine [14]. In contrast, PMSon exposes features that describe the 
data and maps these to sonic parameters. Previous sonifications of molecular simulation data seemed to have favoured PMSon and auditory icons/earcons rather than model-based techniques. This is likely because MD simulations already represent a physical model (see section 2.2) and adapting this dynamical system for the purposes of model-based sonification is challenging.

Rau et. al. [15] demonstrate a PMSon for interrogating features of a static molecule in the Megamol [16] visualization framework. Their approach was to create audio representations of features that are known to be chemically interesting, such as the forming and breaking of hydrogen bonds. Sumo is a plug-in for the Python based molecular simulation environment PyMol [17].This project had the fairly broad aim of providing a general framework for implementing various sonifications within PyMol. A relevant example application created parameterized earcons by mapping the pairwise distances of all atoms of an amino acid onto the parameters of a set of resonant filters. These were offset in both time and space according to their deviation from a reference amino acid. Although no formal studies were undertaken, the designers observed that it was possible to distinguish between them and thus perceive conformational differences in the molecule [18]. Grond et al show a technique for mapping structural information of secondary RNA structures to sonic parameters with a view to aiding browsing and classification; however, there was no attempt to sonically render dynamical walks along free energy surfaces, which is our focus here. [19]. Hermann describes some of the critical issues that arise when designing a PMSon, observing that mappings are not necessarily transparent to a first time listener without some kind of "code book" |20|. This point is reiterated by Wishart when describing the design of his piece, Supernova, which sonified astronomical observations: “...there is no particular reason to use one mapping rather than another. As a result, the sonic outcome would be entirely dependent on the mapping chosen." [21].

\subsection{Molecular representation}

Representational arbitrariness is a particularly interesting issue when it comes to atomistic and molecular representations, owing to the fact that they are too small to be experienced directly, either visually or aurally. There is an inevitable degree of flexibility when designing representations of imperceptible phenomenon, which is evident in the range of available molecular visualisation approaches, e.g., Pauling's paper protein helices [22], Kendrew's metal, wood and plastic protein structures [23], and the increasingly common digital 'ribbon renderings'.[24]. When it comes to sonification, rendering conventions are far from established. Depicting an atom as a sphere is, in some sense, an arbitrary decision, but makes some sense insofar as both are spatially delimited. Attempting to define such a clearly delimited object in the audio realm is not as straightforward, neither spatially nor compositionally. It is difficult to assert what constitutes a single atomistic object in a piece of sound design.

However, there are a wide range of "non-local" properties important in biomolecular science (e.g. potential energy, free energy, electrostatic energy, temperature, conformational state membership, etc.). Such properties are extremely difficult to visualize using conventional rendering strategies (and even if there were effective strategies, would lead to significant visual congestion) owing to their non-locality. We believe that such properties are the most interesting to explore in a sonification context: hence our focus on free energy in this work.
There is a question of the level of intervention that the sonification designer should take. If a dataset is rendered as directly as possible (i.e. converted to audio), then perhaps any audible features present must be features of the data. But this rule may depend on the source and type of data (plus artifacts of the transform). For example, a set of measurements of how temperature changes over time might be treated differently to a non-local parameter that represents the overall instability of a system. In the latter case, it may be necessary to map the data in a less direct way to convey its provenance. Scaletti $[25]$ categorises the directness of mappings through the idea of different orders: for $0^{\text {th }}$ order, the data is directly read as an audio waveform, for $1^{\text {st }}$ order the data is used to modulate a carrier signal. The approach presented here uses many 1st order, one-to-many [26] mappings that attempt to create a certain perceptual effect related to the significance of a given feature. A problem that may be encountered with this approach is that it is atheroetical; the decisions made are based on some subjective sound design process and the results often represent the designer's sensibilities just as much as the underlying data set [27]. The techniques used in this project are primarily parameter mappings, which certainly do encounter some of the issues raised above. Acknowledging these issues is important although addressing them all in detail is out of scope for this paper.

This work extends the practice of molecular sonification by seeking an auditory display of how a molecule walks along a free energy landscape and its relation to fundamental dynamic processes of biomolecules, something that builds on our previous work developing real-time sonification strategies for molecular dynamics simulations ([28, 29|30|). In these prior works, we sonified atomistic systems and events including atomic collisions, atomic clustering, and vibrational spectra. This work focuses on the features of molecular systems. A simulation of the biomolecule Alanine dipeptide (AD) is analysed using both hidden and observed Markov models to extract features of the underlying free energy landscape. These features are then mapped to sonic parameters to generate coupled visual and auditory display of structural and dynamic information respectively.

This paper is organized as follows: section 2 explains some of the underlying physical ideas and the modeling of biomolecular dynamics, section 3 explains our sonification strategy, some details of the implementation are given in section 4 and our conclusions and outlook for further work are given in section 6 An example of the sonification described in this paper can be found at https://vimeo.com/255391814

\section{BIOMOLECULAR CONFORMATIONAL DYNAMICS}

\subsection{Free energy landscape}

Biomolecules such as proteins and nucleic acids are dynamic objects comprised of $n$ atoms, each of which interacts with other atoms in the same molecule and the cellular environment. A molecular system has $3 n$ degrees of freedom: each atom moves in the $x, y$, and $z$ direction. Typically biomolecules are comprised of thousands of atoms, leading to high-dimensional dynamics.

At any given time, a molecule adopts a particular shape, or conformational state. Researchers are typically interested in understanding the networks of conformational states that characterize a particular molecule. Networks of highly connected states in which the system has a relatively long residence time are called metastable states. Of particular interest in many applications is 
understanding how long it takes a molecule to travel between different metastable states. Conformational states are of interest because they are directly linked to the molecule's function, insight [31] which has been verified extensively through experiments [32] and computational studies [33, 34].

Any given conformational state has an associated free energy. Highly probable conformations have a lower free energy than improbable conformations. Rises and falls in free energy as a function of molecular shape (atomic coordinates) define a free energy landscape, which is illustrated in further detail in what follows.

\subsection{Molecular dynamics simulation}

The output of MD simulations are a series of regularly timed snapshots (frames) of atomic configurations as the system evolves, called trajectories. An animated example trajectory can be viewed at https://vimeo.com/255526473. With enough trajectories it is possible to understand the probability that a molecule occupies certain states, and develop a corresponding map of the free energy landscape. The primary challenge in constructing such maps arises from the system's high dimensionality. Researchers are therefore focussing on dimensionality reduction techniques for identifying only those coordinates (or combinations of coordinates) which take the system from one metastable state to another.

\subsection{Markov Models}

Markov state models have found widespread use in recent years as a dimensionality reduction technique to analyze the metastable dynamics of biomolecules [35]. Their popularity stems from their ability to produce predictive and easy to understand results as well as their ability to parallelize the problem of resolving very long timescale processes. Markov models transform a trajectory into a chain of $n$ discrete states. These states are called observed states (or sometimes microstates) and form the data from which both types of Markov model can be estimated. In general we refer to a chain as $x_{t}$ and a specific element by its position in the chain: $x_{2}=3$ denotes that the second frame of the chain is in state 3 . We refer to the set of all possible discrete states as $\mathbf{x}$. There are two related and widespread approaches: observed Markov state models [36] and hidden Markov models [37]. This work uses both.

An observed Markov state model (or simply Markov state model, MSM) assumes the probability of transitioning to observed state $b$ in a time $\tau$ given we are in state $a, P\left(x_{t+\tau}=b \mid x_{t}=a\right)$, only depends on the states $a$ and $b$ and not on the states visited at times $t-1, t-2, \ldots, 0$. This property is known as the Markov property and any chain that satisfies this is known as Markovian. The dynamical information of the MSM is contained within a transition matrix, $\mathbf{T}(\tau)$, whose elements are the conditional transition probabilities, i.e. $T(\tau)_{a, b}=P\left(x_{t+\tau}=b \mid x_{t}=a\right)$.

The primary problem with observed Markov state models is that they contain too much information: the observed states need clustering into a smaller number of metastable states in order to make quantitative predictions about their dynamics. A hidden Markov model (HMM) represents a sort of fuzzy clustering of the observed states into a set of metastable states (or hidden states, $\mathbf{X}$ ), i.e. instead of describing a particular conformation (observed state) as unambiguously belonging to a metastable state, a probability of membership is given. A HMM consists of a transition matrix for the metastable states and a membership matrix $\mathbf{M}$ whose elements are the conditional probability of being in

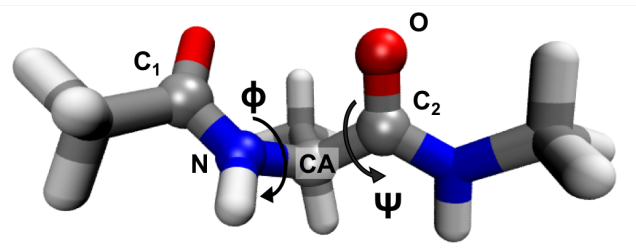

Figure 1: Atomic structure of Alanine dipeptide (AD). The cylinders represent chemical bonds and their intersections represent atoms. Grey, blue, red and white colors are carbon, nitrogen, oxygen and hydrogen atoms respectively. The atoms involved in the $\phi, \psi$ dihedral angles are labeled and highlighted as spheres. The $\phi$ angle is formed from the intersection of the planes formed by the atoms $\left(\mathrm{C}_{1}, \mathrm{~N}, \mathrm{CA}\right)$ and $\left(\mathrm{N}, \mathrm{CA}, \mathrm{C}_{2}\right)$. The $\psi$ angle is formed from the planes formed by the atoms $\left(\mathrm{N}, \mathrm{CA}, \mathrm{C}_{2}\right)$ and $\left(\mathrm{CA}, \mathrm{C}_{2}, \mathrm{O}\right)$.

a metastable state $(A)$ given a particular observed state $(a)$, i.e. $M_{A, a}=P\left(X_{t}=A \mid x_{t}=a\right)$. HMMs work well in describing biomolecular dynamics in the regime where the underlying dynamics are metastable. In other words the proportion of observed states with membership probabilities intermediate between 0 and 1 are small in comparison with the total number of observed states.

\subsection{Alanine Dipeptide Model}

The "hello world" example of a biomolecule exhibiting metastable dynamics is Alanine dipeptide (AD), as shown in figure 1 The metastable dynamics of $\mathrm{AD}$ are reasonably well described with reference to two dihedral angles made by atoms in the peptide bonds [38], the $\phi$ and $\psi$ angles, also shown figure 1] The free energy landscape of AD projected onto these two dimensions is shown in figure 2 A. The light yellow colour denotes free energy wells, i.e. regions with a low value of free energy which define the metastable states. The lighter purple regions are those which are visited only briefly on the way to a metastable well, known as transition regions. As the dihedral angles are periodic, conformations with $\phi / \psi=180^{\circ}$ are equal to those with $\phi / \psi=-180^{\circ}$. This means that rather than a $2 \mathrm{D}$ plane, the free energy landscape actually resides on a torus, i.e. each edge of the chart should be wrapped around to meet the opposite side. For the sake of simplicity we show it here in the form in which it is typically rendered by practitioners in the field.

For the purposes of this paper two models were created - an observed MSM and a HMM. Details of the data and calculations used to generate the models can be found in section 4 The estimated transition matrix for the MSM results in 500 eigenvectors which describe the various relaxation modes of the dynamics. The first eigenvector $\mathbf{q}^{1}$ is equal to the stationary distribution, $\mu(\mathbf{x})$. The next three eigenvectors are slow relaxation modes $\left(\mathbf{q}^{2,3,4}\right)$ which define population transfer between metastable states. The next five eigenvectors $\left(\mathbf{q}^{5-9}\right)$ are fast relaxation modes which define population transfer within metastable states. Each relaxation mode has an associated timescale (the corresponding eigenvalue). The remaining eigenvectors were discarded as the associated timescales for these modes was faster than the time resolution of the data used to estimate the model and so were not considered statistically robust. The HMM was estimated by assuming four metastable states. The results are shown in figure 2 Figure 2 2 shows the metastable state transition matrix, T. Each circle repre- 

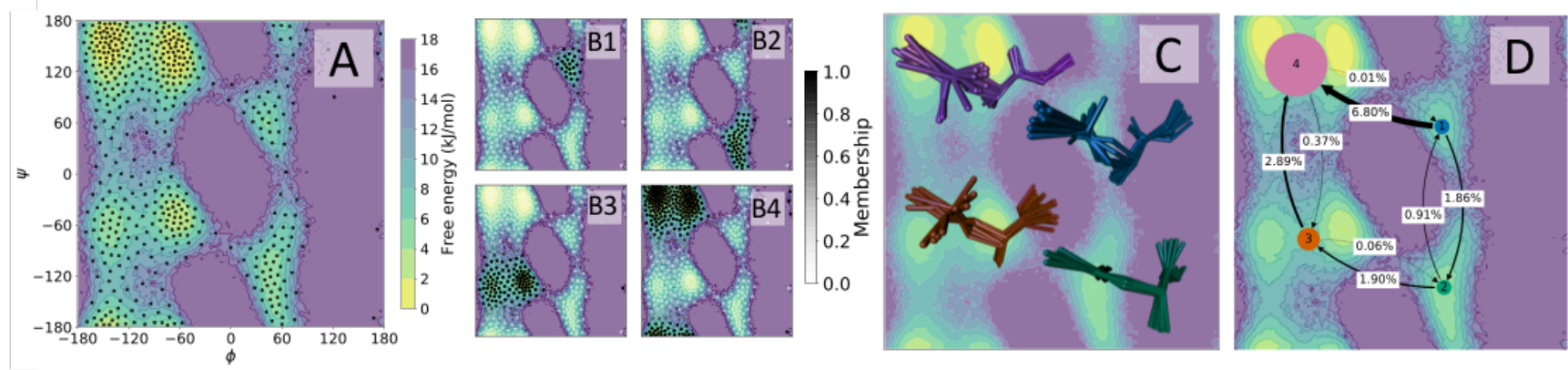

Figure 2: The hidden Markov model for AD. A: The free energy landscape of AD projected onto the peptide $\phi$ and $\psi$ dihedral angles. The lighter the colour the lower the free energy and hence the more stable those atomic configurations. The yellow regions define four metastable basins centered on coordinates $\left(60^{\circ}, 60^{\circ}\right),\left(60^{\circ},-120^{\circ}\right),\left(-120^{\circ},-60^{\circ}\right),\left(-120^{\circ}, 120^{\circ}\right)$. The purple regions are not visited during the simulations used as input for the model. The black circles are the centers of 500 observed states for both the observed and hidden Markov models. Each frame of the trajectory is assigned to the nearest observed state.B1-4: Each panel represents rows 1 - 4 of the membership matrix. Each circle represents an observed state coloured according to its membership probability to each metastable state. C: Ten sampled conformations of each metastable state of AD overlaid over the center of their free energy well. The hydrogen atoms have been removed for clarity. D: The transition matrix and stationary distribution of metastable states. Each circle represents a metastable state with the area of each circle proportional to the stationary distribution. The arrows between each state show possible transitions with the width proportional to the conditional transition probability (also shown in the white boxes on each arrow).

sents a metastable state with the area of the circle proportional to its stationary distribution, $\mu\left(X_{1, \ldots, 4}\right)$. The arrows show the conditional probability of transitioning to each state. For example, the probability of transitioning from state 1 to state 4 is $6.79 \%$. State 4 is by far the most stable, followed by 3,2 and then 1 . As there are no transition regions between state 1 and 3 and between 2 and 4 , the probability of transitions between these pairs of states is zero. Figure 2 $C$ shows an overlay of ten characteristic structures for each metastable state, overlaid over their respective free energy wells. Figures 231-4 show the rows of the membership matrix. Each circle represents one of the observed states, coloured according to the membership probability to each metastable state. The partitioning of the basins is clearly shown by the regions of black circles (high probability of metastable membership) vs. white circles (low probability of metastable membership).

\section{SONIFICATION}

The sonification is designed to convey features of the free energy landscape concurrently alongside visual display of structural information, i.e., a molecular animation from an example trajectory. It therefore requires three objects: a model of AD dynamics, an example trajectory and an animation of the example trajectory. In this work the data used to estimate the model of $\mathrm{AD}$ (the input trajectories) are different to the example trajectory although in principle trajectories from the input data could be used as example trajectories. The following section provides a description of the features derived from the data followed by a description of the components of the sonification and how features of the data are mapped to synthesis parameters.

\subsection{Free Energy Landscape Features}

The features of the data are split into two categories: static and dynamic. Static features are derived from the properties of the AD

\begin{tabular}{l|l|l|l} 
Type & Scope & Parameter & Layer \\
\hline Dynamic & Intra & Free Energy $(F(a))$ & B \\
& & Fast Mode $\left(\left[\mathbf{q}^{i}\right]_{a}\right)$ & $\mathrm{C}$ \\
\cline { 3 - 4 } & Inter & $\begin{array}{l}\text { Shannon Entropy }\left(H_{a}\right) \\
\text { Membership }\end{array}$ & $\mathrm{A}$ \\
& \multirow{4}{*}{---} & \\
& & probability $\left([\mathbf{M}]_{a}\right)$ & $\mathrm{A}$ \\
\cline { 3 - 4 } & & Well min $(U L[h])$ & $\mathrm{A}$ \\
& & Well max $(U B[h])$ & $\mathrm{A}$ \\
& Histogram area $(A[h])$ & $\mathrm{A}$
\end{tabular}

Table 1: Simulation features exposed via OSC. Type refers to whether the features are fixed at time 0 (static) or change at each trajectory frame (dynamic). Scope refers to whether the features relate to transitions between states (inter) or within a state (intra).

molecule and do not change after they are initialised at time 0 . Dynamic features are those derived from each frame (i.e. from each observed state) of the example trajectory as well as the model features. As well as categorizing the features as static or dynamic, a further distinction is drawn between those that relate to changes within the current metastable state (intrastate) and those that pertain to changes between the metastable states (interstate). Table 1 summarizes these two categorizations. This second classification is useful because it draws the distinction between features based on characteristics of the physical dynamics rather than how they were generated. The interstate features represent the most physically important characteristics of the dynamics and free energy landscape and so form the core of the mapping strategy.

Firstly, we discuss static features: these are derived from the shape of the free energy wells associated with each metastable state. Each part of the free energy landscape is assigned a probability of membership to a metastable state so the limits of each well are not well defined. In order to overcome this problem each observed state is assigned to the metastable state for which it has 
the highest probability of membership. The three static features for each metastable state, $U B[h], L B[h], A[h]$ are interstate features. They are principally related to two physical characteristics: the relative stability and conformational flexibility of each metastable state. The focus of the mapping strategy is to find a way to describe them aurally. The free energy for each observed state $a$, $F(a)=-k T \ln \left(\mu\left([\mathbf{x}]_{a}\right)\right)$ was calculated and scaled to lie in the range $(0,1)$. Here $k$ refers to the Boltzmann constant and $T$ to the temperature. The static features for each metastable state were derived from a histogram of the free energies of observed states assigned to that metastable state. We denote the histogram for metastable state $A$ as $h_{A}(F)$ (or $h$ in general). The properties we derive from $h(F)$ are (1) its upper bound, $U B[h]$, (2) its lower bound $L B[h](3)$ its area $A[h]=\int_{0}^{1} \mathrm{~d} F h(F)$. The histograms for states 1 (blue) and 4 (pink) are shown in figure 5 . The model has four metastable states meaning there are $4 \times 3=12$ static features. The upper and lower bounds are related to the free energy well minima and maxima for each metastable state. The area of the histograms is proportional to the overall volume of the free energy well.

Secondly, we discuss dynamic features: these change with each observed state in the example trajectory. For each observed state they are: (1) its probability of membership to each of the four metastable states, (2) the Shannon entropy of its metastable state assignments (3) its absolute free energy, (4) its projection into the five fast relaxation modes. For each observed state there are $4+1+1+5=11$ dynamic features. The membership probability describes the probability that a given observed state can be assigned to a given metastable state. For observed state $a$ there are four membership probabilities given by $\left([\mathbf{M}]_{1, a},[\mathbf{M}]_{2, a},[\mathbf{M}]_{3, a},[\mathbf{M}]_{4, a}\right)$. The information or Shannon entropy is a measure of the degree of certainty with which the assignment of an observed state to a particular metastable state can be made. The Shannon entropy for an observed state $a, H_{a}$ is given by $H_{a}=-\sum_{i=1}^{4}[\mathbf{M}]_{i, a} \ln \left([\mathbf{M}]_{i, a}\right) . \quad H_{a}=0$ indicates the observed state is definitely in one metastable state. $H_{a}=\ln (4) \approx 1.4$ indicates it is equally likely to be in any of the four metastable states. The free energy of observed state $a$, $F(a)$ measures the observed state's global stability. These were the same free energies used in the calculation of the static features in section 3.1. The projection of observed state $a$ onto the $i$ 'th fast relaxation modes is given by $\left[\mathbf{q}^{i}\right]_{a}$. Large oscillations in a projection indicate that the system is relaxing along that mode. The values of these projections were scaled to lie in the range $(-1,1)$. Figure 3 shows the dynamic features for a section of an example trajectory.

\subsection{Sonification Layers}

As the molecule traverses different regions of the free energy landscape the sonification conveys information about its properties with the following layers in the audio stream: (A) a continuous pad sound representing membership of different metastable states and their properties, (B) a pulse sound representing the stability of the system and (C) a set of synthesized tones that represent how the system is changing within each metastable state.

The Pad Sound (A) was constructed as follows:

1 Representation: The pad sound represents features of the well in the free energy landscape corresponding to the current metastable state assignment.

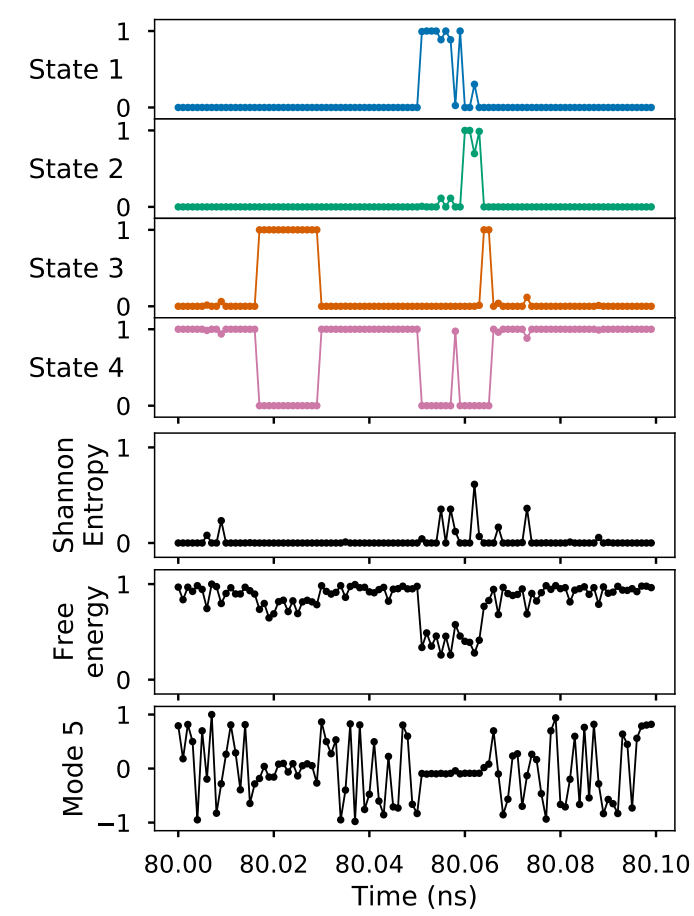

Figure 3: The dynamic features of the model. Each panel shows how a model features varies over 0.1 ns (100 frames) of the example trajectory. State 1-4 show the membership probability of each frame's observed state to each metastable state. The values in each frame across all four panels sum to 1 . Shannon entropy measures the uncertainty of the assignment of each observed state to the metastable states. High entropy indicates an observed state could be considered to belong to more than one metastable state. Free energy is the free energy of each observed state, the lower the free energy the more stable the state. Mode 5 is the first fast relaxation mode which redistributes population within a metastable state. The other four fast modes are not shown.

2 Synthesis: Created using a subtractive, polyphonic synthesiser with noise as a source signal. The basic signal flow of a single voice is shown in Figure 4. The main parameters are the $Q$ value of the resonant filter, the depth and rate of frequency modulations for each voice and the amplitude of each voice.

3 Mapping: Each metastable state is characterised by a set of notes referred to as a note cluster. This design arises from the desire to represent metastable state transitions by tonal groupings, a musical device that non-trained listeners should be able to perceive. The maximum note range is predefined to three octaves. The relative values of $A[h]$ for each metastable state defines the number of notes in its corresponding note cluster, these notes are then evenly distributed between the lowest and highest notes. The values of $L B[h]$ and $U B[h]$ define the lowest and highest notes of each cluster respectively. This is shown in figure 5 for metastable states 1 and 4 . State 4 (pink) has a smaller range, but a larger area resulting in a dense, tightly spaced note cluster at the lower extent of the note range. State 1 (blue) has a large range but small area resulting in a more sparse note cluster. State 4 has its lower bound below that of state 1 and so 


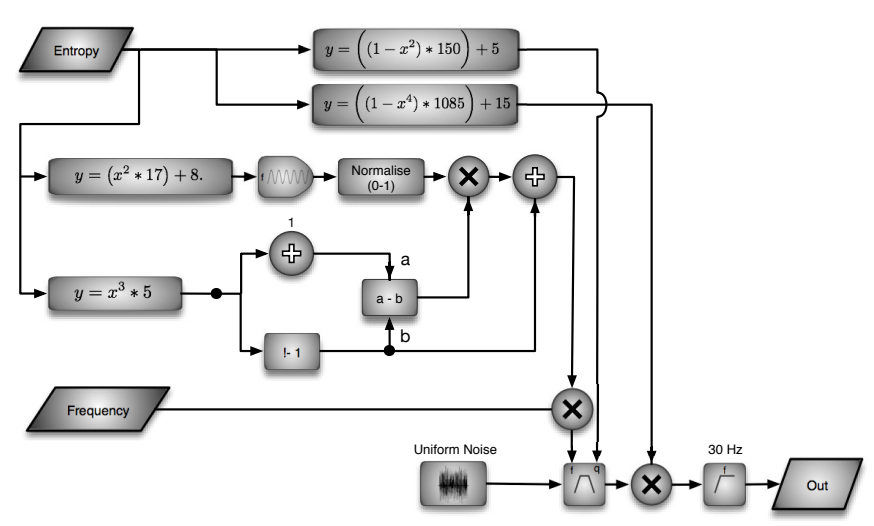

Figure 4: Single synthesis voice as used for each note of a metastable state note cluster. The set of multiple instances (one per note) form the pad sound. The inputs are the frequency of each note in the note cluster and the entropy. The note cluster is determined by the membership probability values at the current frame.

the lowest note of the cluster is below that of state 1. An accepted limitation of this mapping is that it does not attempt to classify the clusters in terms of their harmonic relationships. Instead, it deals with them as distributions of notes within a range, with a given extent and density. It may be possible to create a hierarchy of tonal groupings from which to choose but this is highly genre specific. This is an outstanding issue in that listeners may interpret the harmonic relationship of two note clusters as significant when this is not intended as part of the mapping (e.g. stacked whole tones vs. stacked fourths). The membership probabilities (M) control the choice of note cluster by linearly interpolating between the values defined by the static parameters of each state. This means that if $[\mathbf{M}]_{1, a}=1$ then the cluster defined by the static properties of metastable state 1 will be used. In the case that $[\mathbf{M}]_{1, a}=[\mathbf{M}]_{2, a}=0.5$ then the lowest and highest notes will lie halfway between those defined by the static parameters of state 1 and 2. Large values of the Shannon entropy $(H)$ represent observed states which could be assigned to more than one metastable state, physically this means they are in transition regions between two metastable states. $H$ is mapped to the width and rate of frequency modulations of the voices for the note clusters as well as the bandwidth of the filter such that it tends toward noise for higher values of $H$. This is a one-to-many mapping that is designed to create a perceptual effect of instability. $H$ tends to remain at 0 with occasional spikes as the system transitions. This is shown in figure 3 where there are spikes between 80.05 and 80.06 nanoseconds as transitions occur between all four states. These spikes, although fleeting, are important features. To emphasise transitions an asymmetric smoothing function is employed such that $H$ values increase quickly but decrease slowly.

The Pulse sound (B) was constructed as follows:

1 Representation: The overall stability of the current observed state in relation to the global minimum. The pulse sound is designed to underpin the entire sonification. The rationale here is that a rhythmic kick drum sound is commonly used as a rhythmic marker upon which all other elements are constructed. This mirrors the way in which the free energy landscape underpins the dynamics of the physical system.

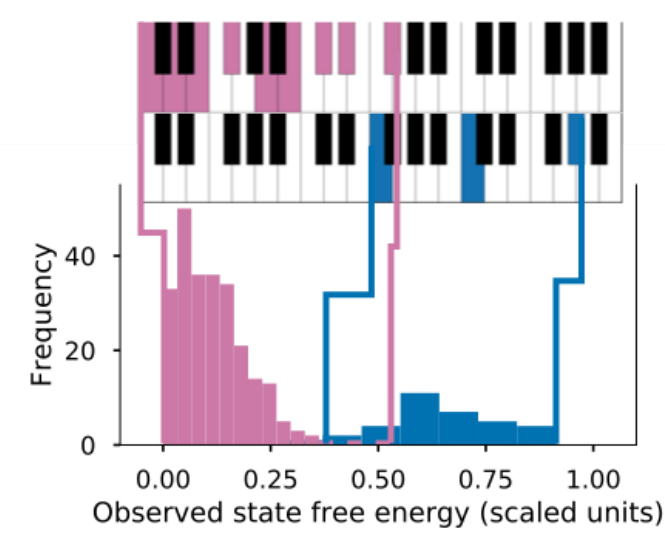

Figure 5: The mapping of static properties of the metastable states to note clusters. Each of the observed states was first assigned to exactly one metastable state. The bottom chart shows the distribution of free energies of observed states which have been assigned to the metastable states 1 (blue) and 4 (pink). States 2 and 3 are not shown for clarity. The coloured notes of the keyboard are the note clusters used to represent the metastable states. The relative upper and lower bounds of the distributions determine the highest and lowest notes in the cluster (as shown by the vertical connectors). The relative area of each distribution determines the number of notes in the note cluster. The ratio of the areas of state 4's distribution to state 1 's distribution is approximately $3: 1$. This determines the $9: 3$ ratio of the number of notes in each note cluster.

2 Synthesis: Generated by sweeping the frequency of a sine wave from approximately $130 \mathrm{~Hz}$ to $20 \mathrm{~Hz}$ over a short period (100-550ms) and passing it through a hard clipping function and stereo delay line. The relevant parameters are the rate at which the sweep is triggered, the length of time the sweep takes, the amount of drive in the hard clipping function and the amount of feedback in the delay path.

3 Mapping: The synthesis parameters are mapped to the absolute free energy $F\left(x_{t}\right)$. The variable is inverted and filtered logarithmically. The filter settings are slightly different for each synthesis parameter but, in general terms, act to allow fast increases in its values and slow decreases to ensure the temporal alignment between visual and sonic representations. The $\left(F\left(x_{t}\right)\right)$ of each observed state measures its stability relative to the global free energy minimum. This feature allows the sonification to draw a distinction between an observed state being globally unstable and yet part of a relatively stable metastable state (or vice versa).

Within-Metastable State Tones $(\mathbf{C})$ were constructed as follows:

1 Representation: The extent to which the system is relaxing along one of the fast modes. This gives an impression of high frequency movements of the molecule that are not represented by the membership probabilities.

2 Synthesis: The fast mode projections are oscillatory signals between -1 and 1 and are exported as PCM wav files (a $0^{\text {th }}$ order mapping in Scaletti's terminology [25]). At the $20 \mathrm{~Hz}$ framerate being used for the animation the oscillations are subsonic. Scanned synthesis is used in order to render the content as audible [39. The relevant parameter is the audio file that is used as the wavetable to be scanned. Other parameters that affect the sound are the window size and the scanning frequency. 
3 Mapping: Scanned synthesis was developed to allow for the direct manipulation of synthesis timbre using a physical model and represents an extension of wavetable synthesis. In this case it allows for a rolling window of the audio buffer to be scanned at a given frequency, with the effect that increased frequency and amplitude of oscillation in the window results in a brighter timbre. These can be heard panned from hard left (mode $\mathbf{q}^{5}$ ) to hard right (mode $\mathbf{q}^{9}$ ). The scanning frequency is defined by the lowest note of the set of note clusters (effectively the root of the most stable state).

\section{IMPLEMENTATION}

The simulation data was taken from a publicly available repository which accompanies the paper [38] found at https://simtk. org/projects/alanine-dipeptide/ A full explanation of the methods used to generate the data are given in the paper. All modelling was done using the Python 3.5 programming language, the Markov models were generated by PyEMMA 2.4 [40]. Both the HMM and MSM used the same set of 500 observed states and each were estimated with a lag time of $\tau=1.0 \mathrm{ps}$. The HMM was estimated by specifying four metastable states. This number was chosen so that the approximation of metastability was most accurate at the lag time used. The molecular animations were generated using VMD [24]. A 500ns long example trajectory was used in the sonification. This was generated from the HMM, rather than using an input trajectory as they were all of insufficient length to sample each metastable state regularly. While this trajectory does not strictly obey the original equations of motion used to generate the input data it reproduces all the modelled features (transition probabilities, relaxation time-scales, stationary distribution) and so is indistinguishable from a trajectory generated using the original equations of motion. A Python script, using the package OSC 1.6.4, was used to create a client which sent the static and dynamic parameters as messages to the audio processing software. The messages were sent using the OSC protocol. The dynamic parameters were sent at a rate of 20 trajectory frames per second (corresponding to a ratio of $20 \mathrm{ps}$ to $1 \mathrm{~s}$ simulation to physical time). The audio processing was implemented in Max/MSP.

Everything required to reproduce this work (except the input data which can be downloaded separately) can be found at https://osf.io/rzp3k/?view_only= b5802dfce6da4dd59dfb6b406ae033f0.

\section{EXAMPLE SONIFICATION}

The example provided (link in sec. 1) shows an animation of an excerpt of trajectory data for Alanine Dipeptide accompanied by our sonification. This section provides a description of the audible features and how they relate to the mapping strategy. The pad layer (A) provides information about the structure of the free energy landscape for the configuration at a given frame; it can be heard ramping between the note clusters defined by the static features of the four metastable states. It stays primarily in state 4 , as this is the most stable. At 00:13 the animation shows a significant change in the dihedral angles; this is linked to a change in the metastable state and a shift to a different well on the free energy landscape, which is depicted by the change in note cluster of the pad sound. The noise/wind effect that can be heard at 00:10 is caused by an increase in the Shannon entropy and gives an indication that there is some uncertainty in the assignment of the current observed state. The pulse/kick layer (B) is correlated to the absolute free energy; at 00:10, the current metastable state has a lower absolute free energy and the rate, level of distortion and delay feedback are increased resulting in the sound becoming more prominent. The layer representing the fast relaxation modes (C) can be heard as the metallic, buzzing sounds panned across the sound-field. The scanned synthesis method means that amplitude and frequency of activity in the data is correlated to density of spectra and amplitude of the synthesiser. So an increase in brightness of this sound on the left of the sound-field indicates that the molecule is relaxing along the first of the five modes used.

\section{CONCLUSIONS AND FUTURE WORK}

We have presented a strategy for representing features of the dynamics and the free energy landscape of Alanine Dipetide with sound. This auditory display can be used in tandem with visual display techniques to help build an understanding of how the physical structure of $\mathrm{AD}$ relates to the underlying free energy landscape and the resulting dynamic processes. Future user studies with domain experts will allow an assessment of the efficacy of the sonification; a sketch for this would entail participants viewing an animation of a simulation trajectory with and without the various layers of the sonification to assess if and how they show an increased appreciation of the free energy dynamics. In the future we hope to extend this implementation to allow a degree of interactivity in manipulating the example trajectory. Initially, this would take the form of allowing the user to manipulate the playback position, speed and loop points of the trajectory. This would allow them to focus on regions of interest in the dynamics. In the long term, allowing the user to manipulate the example trajectory using interactive molecular dynamics (for example, NanoSimbox [41]) and hear the resultant sonic effects is an exciting prospect and opens up the possibility of using the system as an instrument for musical expression as well as data exploration.

\section{ACKNOWLEDGMENT}

David Glowacki acknowledges funding from the Royal Society (UF120381) and the Leverhulme Trust. Robert Arbon is funded by the Royal Society (RG130510). Alex Jones is supported by a studentship from the EPSRC and Interactive Scientific Ltd. Lars Bratholm acknowledges EPSRC grant EP/P021123/1.

\section{REFERENCES}

[1] R. P. Feynman, et al., The Feynman lectures on physics. Addison-Wesley, 1963.

[2] M. Hashimoto, et al., "Role of Protein Aggregation in Mitochondrial Dysfunction and Neurodegeneration in Alzheimer's and Parkinson's Diseases," NeuroMolecular Medicine, vol. 4, pp. 21-35, 2003.

[3] P. Singh, et al., "Linking Protein Motion to Enzyme Catalysis," Molecules, vol. 20, no. 1, pp. 1192-1209, 2015.

[4] S. A. Adcock and J. A. McCammon, "Molecular dynamics: Survey of methods for simulating the activity of proteins," Chemical Reviews, vol. 106, no. 5, pp. 1589-1615, 2006.

[5] D. Antoniou, et al., "Computational and Theoretical Methods to Explore the Relation between Enzyme Dynamics and Catalysis," Chemical Reviews, vol. 106, no. 8, p. 3170, 2006. 
[6] M. W. van der Kamp, et al., "Biomolecular simulation and modelling: status, progress and prospects," J. Royal Society Interface, vol. 5, no. Suppl 3, p. 173, 2008.

[7] J. E. Stone, et al., "Gpu-accelerated molecular modeling coming of age," J. Molecular Graphics and Modelling, vol. 29 , no. 2 , p. 116,2010

[8] P. Eastman, et al., "Openmm 4: A reusable, extensible, hardware independent library for high performance molecular simulation," J. Chemical Theory and Computation, vol. 9, no. 1, p. 461, 2013.

[9] D. Case, et al., "Amber 2017," University of California, San Francisco, 2017.

[10] M. Álvarez-Moreno, et al., "Managing the computational chemistry big data problem: The iochem-bd platform," $J$. Chemical Information and Modeling, vol. 55, p. 95, 2015.

[11] B. J. Alder and T. E. Wainwright, "Studies in molecular dynamics. i. general method," J. Chemical Physics, vol. 31, no. 2, p. $459,1959$.

[12] P. W. Atkins and J. de Paula., Atkins' physical chemistry, 10th ed., 2014.

[13] G. Prez-Hernndez, et al., "markovmodel/molPX: More PyEMMA integration through handling of TICA objects," Mar. 2017. [Online]. Available: https://doi.org/10.5281/ zenodo.437707

[14] T. Hermann and H. Ritter, "Listen to your Data: ModelBased Sonification for Data Analysis," in Advances in intelligent computing and multimedia systems. Inst. for Advanced Studies in System research and cybernetics, 1999, pp. 189-194.

[15] B. Rau, et al., "Enhancing visualization of molecular simulations using sonification," in 1st International Workshop on Virtual and Augmented Reality for Molecular Science. IEEE, March 2016, pp. 25-30.

[16] S. Grottel, et al., "MegaMol-A prototyping framework for particle-based visualization," IEEE Transactions on Visualization and Graphics, vol. 21, pp. 201-214, February 2015.

[17] “Pymol," 2017. [Online]. Available: https://pymol.org/2/

[18] F. Grond and F. Dall Antonia, "Sumo. A Sonification Utility for Molecules," in Proceedings of ICAD 2008, no. $14 . \quad$ International Community for Auditory Display, 2008, pp. 1-7.

[19] F. Grond, et al., "Browsing RNA Structures by Interactive Sonification," in ISon, 3rd Interactive Sonification Workshop, Stockholm, 2010, pp. 11-16.

[20] T. Hermann, et al., "Sonification of markov chain monte carlo simulations," in ICAD 2001. Espoo, Finland: Georgia Institute of Technology, 2001, pp. 208-216.

[21] T. Wishart, "From Sound Morphing to the Synthesis of Starlight. Musical experiences with the Phase Vocoder over 25 years," Musica/Tecnologia, vol. 7, 2013.

[22] L. Pauling, et al., "The structure of proteins: Two hydrogenbonded helical configurations of the polypeptide chain," $P$. National Academy of Sciences, vol. 37, no. 4, p. 205, 1951.

[23] J. C. Kendrew, et al., "A three-dimensional model of the myoglobin molecule obtained by x-ray analysis," Nature, vol. 181, p. 662, 031958.
[24] W. Humphrey, et al., "VMD - Visual Molecular Dynamics," Journal of Molecular Graphics, vol. 14, pp. 33-38, 1996.

[25] C. Scaletti, "Sound synthesis algorithms for auditory data representations," in Auditory Display: Sonification, Audification, And Auditory Interfaces, G. Kramer, Ed. Reading: Addison-Wesley, 1994.

[26] A. Hunt, et al., "The Importance of Parameter Mapping in Electronic Instrument Design," Journal of New Music Research, vol. 32, no. 4, pp. 429-440, 2003.

[27] E. Murphy, et al., "A Semiotic Approach to the Design of Non-speech Sounds," in Haptic and Audio Interaction Design, D. McGookin and S. Brewster, Eds. Berlin, Heidelberg: Springer Berlin Heidelberg, 2006, pp. 121-132.

[28] D. R. Glowacki, et al., "A gpu-accelerated immersive audiovisual framework for interaction with molecular dynamics using consumer depth sensors," Faraday Discuss., vol. 169, pp. 63-87, 2014.

[29] T. Mitchell, et al., "danceroom spectroscopy: At the frontiers of physics, performance, interactive art and technology." Leonardo, vol. 49, no. 2, pp. 138-147, 2016.

[30] D. Glowacki, "Sculpting molecular dynamics in real-time using human energy fields," in Molecular Aesthetics, P. Weibel and L. Fruk, Eds. Cambridge, MA: The MIT Press, 2013, pp. 248-257.

[31] H. Frauenfelder, et al., "The energy landscapes and motions of proteins," Science, vol. 254, no. 5038, p. 1598, 1991.

[32] Y. Santoso, et al., "Conformational transitions in dna polymerase i revealed by single-molecule fret," $P$. National Academy of Sciences, vol. 107, no. 2, p. 715, 2010.

[33] D. Wales, Energy Landscapes: Applications to Clusters, Biomolecules and Glasses, ser. Cambridge Molecular Science. Cambridge University Press, 2004.

[34] N.-V. Buchete and G. Hummer, "Coarse master equations for peptide folding dynamics," J. Physical Chemistry B, vol. 112, no. 19, p. 6057, 2008, pMID: 18232681.

[35] J. D. Chodera and F. Noé, "Markov state models of biomolecular conformational dynamics," Current Opinion in Structural Biology, vol. 25, pp. 135 - 144, 2014.

[36] J.-H. Prinz, et al., "Markov models of molecular kinetics: Generation and validation," The Journal of chemical physics, vol. 134, no. 17, p. 174105, 2011.

[37] F. Noé, et al., "Projected and hidden markov models for calculating kinetics and metastable states of complex molecules," The Journal of chemical physics, vol. 139, no. 18, p. 184114, 2013.

[38] J. D. Chodera, et al., "Automatic discovery of metastable states for the construction of markov models of macromolecular conformational dynamics," J. Chemical Physics, vol. 126, no. 15, p. 155101, 2007.

[39] B. Verplank and R. Shaw, "Scanned Synthesis," The Journal of the Acoustical Society of America, vol. 109, no. 5, 2001.

[40] M. K. Scherer, et al., "PyEMMA 2: A Software Package for Estimation, Validation, \& Analysis of Markov Models," J. Chemical Theory \& Computation, vol. 11, p. 5525, 2015.

[41] M. O. Connor, et al., "Sampling molecular conformations and dynamics in a multi-user virtual reality framework," ArXiv e-prints, Jan. 2018, ArXiv:1801.02884. 